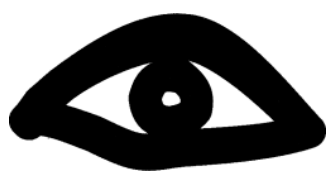

REVISTA DE ESTUDIOS E INVESTIGACIÓN

EN PSICOLOGÍA Y EDUCACIÓN

eISSN: 2386-7418, 2017, Vol. Extr., No. 11

DOI: https://doi.org/10.17979/reipe.2017.0.11.2454

\title{
Perceção/atitudes dos pais de alunos com NEE face à inclusão Perception / attitudes of parents of SEN students in relation to inclusion
}

\author{
*Maria Celeste Sousa Lopes, **Ana Marques, ** Bruno Marques, **Claudia Salgado \\ * IESFAFE/ UPT, ** MEC/ iesfafe
}

\begin{abstract}
Resumen
Atualmente verifica-se uma evolução do sistema educativo português, no sentido de tornar as escolas públicas verdadeiras comunidades educativas de inclusão, onde todos os alunos têm iguais oportunidades de aprendizagem. O papel da familia no processo de inclusão dos filhos com necessidades educativas especiais é crucial. É quem melhor conhece a criança, possuindo uma informação valiosa que os professores deverão ter em conta na hora de planificar e adequar as estratégias educacionais. A presente comunicação resulta de um estudo conduzido com o intuito de conhecer as percepções/atitudes dos pais com filhos com necessidades educativas especiais face a uma educação inclusiva.

Palavras chave: inclusão, atitudes, percepção, NEE, pais
\end{abstract}

\section{Abstract}

Currently there is an evolution of the Portuguese education system, in the sense of making public schools true inclusion educational communities, where all students have equal opportunities for learning. The role of the family in the process of including children with special educational needs is crucial. It is the one who best knows the child, possessing valuable information that teachers should take into account when planning and adjusting educational strategies. This communication is the result of a study conducted to understand the perceptions / attitudes of parents with children with special educational needs facing an inclusive education.

Keywords: Inclusion, attitudes, perception, SEN, parents

\section{Introdução}

Gradualmente tem-se assistido a uma reestruturação do sistema educativo no sentido de existir uma inclusão de crianças e jovens com Necessidades Educativas Especiais no ensino regular. Com a Declaração de Salamanca (UNESCO, 1994) uma educação para todos, onde todos os alunos, independentemente das suas necessidades e caraterísticas, possam aprender juntos, tem vindo a ser alvo de reflexão por parte de entidades internacionais, que reconhecem a necessidade e a urgência de garantir a educação para as crianças, jovens e adultos com NEE no quadro do sistema regular de educação.

A filosofia de uma escola inclusiva tem uma visão de que uma turma regular de uma escola de ensino regular é o local ideal para as aprendizagens do aluno com necessidades educativas especiais, uma vez que será nesse contexto, ou seja, na companhia dos seus colegas sem NEE, que a criança/jovem irá encontrar o melhor ambiente de aprendizagem e de socialização, como refere Correia (2013).
Claro que uma reestruturação tão profunda no sistema educativo levanta uma série de questões, principalmente na forma como se vai acolher e envolver estas crianças e adolescentes em todas as atividades de aprendizagem escolar, num processo que exige uma adaptação de todo o contexto, envolvendo uma série de intervenientes: a comunidade escolar, a sociedade e os país.

Segundo Palmer, S; Borthwick-Duffy, A \& Widaman, K. (1998 cit. in Matos, Novais, Magalhães \& Dias, 2015) discutiu-se e discute-se muito sobre a inclusão entre os agentes educativos, no entanto, parece existir pouca investigação sobre as perceções dos pais/família sobre o movimento inclusivo.

A família é considerada uma das unidades sociais de base, isto é, a família constitui um dos pilares da vida em sociedade, sendo um elemento fundamental na vida e no desenvolvimento da criança. Assim, a escola, instituição considerada como dos grandes agentes de socialização logo a seguir à instituição familiar, deverá incluir a família nas decisões mais importantes respeitantes à criança, quer sejam crianças com um desenvolvimento normal quer sejam crianças com necessidades educativas especiais.

Segundo Correia (2013), os pais são elementos fundamentais na planificação, execução e avaliação dos programas de intervenção dos seus filhos.

De acordo com Barbosa, A; Rosini, C \& Pereira, A (2007 cit. in Matos, Novais, Magalhães \& Dias, 2015)

O papel dos pais é essencial enquanto coconstrutores do sistema educativo, a nível político e social, seja através do seu envolvimento e na complementaridade que podem ter na intervenção educativa, na forma como transmitem mensagens para a escola e para os seus filhos, podendo influenciar as percepções que estes têm sobre os seus pares (p. 445)

Dessa forma, as mensagens que os pais transmitem, sejam explícitas ou implícitas, as atitudes e os comportamentos manifestados pela família nuclear são cruciais para que ocorra uma inclusão educativa com sucesso, devido à influência nas atitudes e nos comportamentos dos seus filhos e nas respostas de intervenção por parte das escolas e dos agentes educativos. Como referem Dessen e Polonia (2007) a integração entre família e a escola traz benefícios para o desenvolvimento da pessoa uma vez que permite que ações conjuntas sejam flexibilizadas e as possibilidades de aprendizagem sejam maximizadas. 
Sendo os pais as pessoas que melhor conhecem as necessidades dos seus filhos, torna-se essencial o envolvimento parental na promoção de uma escola inclusiva.

\section{Método \\ Justificação do estudo}

$\mathrm{Na}$ revisão de literatura, verifica-se que a perceção/atitudes dos pais face à inclusão dos filhos portadores de deficiência é, ainda, uma área pouco estudada, sendo escassos os trabalhos relativos a este tema. Como refere Correia (2013, p. 155), "Em Portugal, o envolvimento parental ainda parece andar pelo consciencializar quer os profissionais, quer os pais da importância da participação destes na educação da criança com NEE." Por conseguinte, e atendendo a uma necessidade de uma escola cada vez mais inclusiva, para além de se considerar fundamental o papel dos pais na educação dos alunos com necessidades educativas especiais, propôs-se investigar a perceção/atitudes dos pais relativamente à inclusão dos filhos, portadores de deficiência, numa escola de ensino regular.

\section{Objetivos do estudo}

O objetivo geral desta investigação foi perceber o envolvimento parental na educação dos filhos com NEE. Mais especificamente pretendeu-se: i) Analisar a percepção/atitudes dos pais no que respeita a uma educação inclusiva; ii) Identificar a motivação dos pais para a inclusão dos filhos com NEE nas turmas de ensino regular; iii) Abordar as expectativas dos pais relativamente a uma educação inclusiva dos filhos; iv) Determinar se o envolvimento parental e as perceções/atitudes dos pais em relação a uma escola inclusiva está associada a variáveis sociodemográficas.

\section{Hipóteses Exploratórias}

Atendendo aos objetivios pretendidos e às questões levantadas formularam-se algumas hipótesis que serviram de fio condutor a toda a investigação.

As hipóteses exploratórias do presente estudo foram as seguintes: Hipótese 1- Dependendo da gravidade das necessidades educativas dos filhos, os pais experienciam diferentes atitudes face à inclusão; Hipótese 2- As mães experienciam uma atitude mais favorável à inclusão dos seus filhos do que os país; Hipótese 3- Os pais de crianças NEE com idades mais avançadas experienciam uma atitude negativa face à inclusão que os pais de crianças NEE com idades mais novas; Hipótese 4- Os pais de crianças NEE com baixo nível de escolaridade experienciam uma atitude negativa face à inclusão que os pais de crianças NEE com nível de escolaridade mais alto.

\section{Variáveis e sua operacionalização}

No presente estudo as variáveis independentes (que di-

zem respeito às dimensões ou caraterísticas manipuladas pelo investigador) foram as seguintes: i) Grau de parentesco: operacionalizada em duas categorias (mãe e pai);i i) Idade dos pais: operacionalizada em três categorias (<= 35 anos; 36-40 anos; >40 anos); iii) Nível de escolaridade dos pais: operacionalizado em três categorias (alto, médio, baixo);iv) Gravidade das necessidades educativas especiais: operacionalizada em duas categorias (significativas e ligeiras).

As variáveis dependentes (sãos as variáveis medidas e observadas pelo investigador, ou seja, surgem ou mudam devido à influencia das variáveis independentes) deste estudo são as percepções e as atitudes dos pais.

\section{Participantes}

Colaboraram no estudo 30 pais. Com filhos com NEE de 3 agrupamentos de escolas e duas Instituições A maioria era do género feminino $(53.0 \%, \mathrm{n}=16)$.A média de idades das mães era de 40.97 anos $(\mathrm{DP}=2.85)$ e a dos pais de 42.50 anos (DP = 3.83). Em termos de habilitações académicas, a maioria das mães e dos pais tinha o ensino secundário (53.3\% e $56.7 \%$ ). As mães com o ensino superior representavam $33.3 \%$ e os pais com o ensino superior representavam $20.0 \%$. A maioria tinha apenas 1 filho (70.0\%). A distribuição das problemáticas das crianças pode ser apreciada na tabela $\mathrm{nr}^{\circ} 1$. As mais referidas foram Dificuldades de aprendizagem (20.0\%), Hiperatividade e défice de atenção (16.7\%) e Deficiência motora (13.3\%). Em $76.7 \%$ dos casos o filho com NEE era o primogénito. A maioria das crianças era do género feminino $(56.7 \%)$.

Tabela

Problemáticas

\begin{tabular}{|c|c|c|}
\hline & $\begin{array}{l}\text { Frequê } \\
\text { ncia }\end{array}$ & $\begin{array}{l}\text { Percentag } \\
\text { em }\end{array}$ \\
\hline Atraso cognitivo & 2 & 6.7 \\
\hline Deficiência intelectual & 1 & 3.3 \\
\hline Deficiência motora & 4 & 13.3 \\
\hline $\begin{array}{l}\text { Dificuldades } \\
\text { aprendizagem }\end{array}$ & 6 & 20.0 \\
\hline Dislexia & 2 & 6.7 \\
\hline $\begin{array}{l}\text { Hiperatividade e défice de } \\
\text { atenção }\end{array}$ & 5 & 16.7 \\
\hline $\begin{array}{l}\text { Perturbações do Espectro do } \\
\text { autismo }\end{array}$ & 2 & 6.7 \\
\hline Síndrome de Apert & 1 & 3.3 \\
\hline Síndrome de Down & 3 & 10.0 \\
\hline Síndrome de Koolen de Vries & 2 & 6.7 \\
\hline Síndrome de Rubienstein & 2 & 6.7 \\
\hline Total & 30 & 100.0 \\
\hline
\end{tabular}

Em termos de gravidade da problemática, em metade dos alunos era grave e em $43.3 \%$ era do tipo ligeira..A média de idades das crianças era de 13.0 anos (DP = 2.82), variando entre um mínimo de 11 anos e um máximo de 18 anos.

\section{Instrumentos}

Este estudo foi realizado com recurso a um questionário com duas partes. Com a parte I, pretendeu-se recolher os dados sociodemográficos, como: grau de parentesco, 
idade, nível de escolaridade e grau das necessidades educativas especiais dos filhos.

Na segunda parte foi utilizado uma adaptação do Inventário Geral de Atitudes quanto à Educação Inclusiva (IGAEI) de Barbosa, Rosini \& Pereira (2007).Segundo estes autores o instrumento tem como intuito avaliar as atitudes dos pais em relação à educação inclusiva, abrangendo crenças e sentimentos perante a inclusão escolar.

\section{Procedimentos}

Os instrumentos foram administrados individualmente nos diferentes estabelecimentos de ensino. Durante a sua administração enfatizou-se a relevância dos seguintes aspetos: 1 - participação da mãe/pai; 2- a não existência de respostas certas ou erradas; 3- a precaução para não omitir nenhuma resposta e 4- o facto do tratamento dos dados ser anónimo e confidencial.

A duração média da administração dos instrumentos foi de 10 minutos por mãe/pai, no entanto existiram mães e pais que recusaram preencher os questionários na presença dos autores do estudo, alegando pouco tempo para a sua realização. Nestas situações, os questionários foram preenchidos em casa e aquando na nova vinda ao estabelecimento de ensino eram devolvidos e esclarecidas quaisquer duvidas ou dificuldades que as mães/pais pudessem ter.

Os instrumentos utilizados foram adaptados dos instrumentos publicados. $\mathrm{O}$ questionário Sociodemográfico foi elaborado pelos autores do estudo. É constituído por 6 (seis) itens. Para responderem aos itens utilizou-se perguntas fechadas.

A análise estatística envolveu medidas de estatística descritiva (frequências absolutas e relativas, médias e respetivos desvios-padrão) e estatística inferencial. O nível de significância para aceitar ou rejeitar a hipótese nula foi fixado em $(\alpha) \leq 0,05$. Na análise inferencial usouse o coeficiente de consistência interna Alfa de Cronbach, o coeficiente de correlação momento produto de Pearson, o teste $t$ de Student para amostras independentes (quando se comparou dois grupos em variáveis de tipo quantitativo), o test $t$ de Student para uma amostra (quando se comparou a média das respostas com uma média teórica) e o teste de Wilcoxon (quando se comparou a mediana das respostas com uma mediana teórica). Os pressupostos do teste $t$ de Student para amostras independentes, nomeadamente o pressuposto de normalidade de distribuição e o pressuposto de homogeneidade de variâncias foram analisados com os testes de Shapiro Wilk e teste de Levene.

A análise estatística foi efetuada com o SPSS (Statistical Package for the Social Sciences) versão 22.0 para Windows

A consistência interna dos instrumentos utilizados nesta investigação foi analisada com o coeficiente de consistência interna Alfa de Cronbach. O valor obtido para o Inventário Geral de Atitudes quanto à Inclusão Escolar foi de .891 (bom). A categorização dos valores do Alfa segue o publicado na literatura.

\section{Resultados}

\section{Análise Diferencial - Hipóteses}

Os pais de filhos com deficiência ligeira apresentam atitudes significativamente mais favoráveis à inclusão do que os pais de filhos com deficiência grave (3.69 vs 3.40), $\mathrm{t}(26)=2.406, p=.026$. conforme se pode ver na tabela 2. O que confirma a hipótese 1 - "Dependendo da gravidade das deficiências dos filhos, os pais experienciam diferentes atitudes face à inclusão".

Tabela 2

Significância da diferença: tipo de gravidade

\begin{tabular}{ccccccc}
\hline & \multicolumn{2}{c}{ Ligeira } & & \multicolumn{2}{c}{ Grave } & \\
\cline { 2 - 3 } \cline { 5 - 6 } Inclusão & $\mathrm{M}$ & $\mathrm{DP}$ & & $\mathrm{M}$ & $\mathrm{DP}$ & $\mathrm{t}$ \\
\hline \multirow{2}{*}{ 3.69 } & 0.29 & 3.40 & 0.35 & $\begin{array}{c}2.406 \\
*\end{array}$ \\
\hline & $* p \leq .05$ & & & &
\end{tabular}

Relativamente à hipótese 2 - “As mães experienciam uma atitude mais favorável à inclusão dos seus filhos do que os pais". Pode-se concluir e, conforme se observa na tabela 3, as mães apresentam atitudes mais favoráveis à inclusão do que os pais (3.59 vs 3.48), embora a diferença não seja estatisticamente significativa, $\mathrm{t}(28)=$ $0.920, p=.366$.

Tabela 3

Significância da diferença: pais vs mães

\begin{tabular}{ccccccc}
\hline & \multicolumn{3}{c}{ Mãe } & & \multicolumn{3}{c}{ Pai } & \\
\cline { 2 - 5 } \cline { 5 - 6 } & $\mathrm{M}$ & $\mathrm{DP}$ & & $\mathrm{M}$ & $\mathrm{DP}$ & $\mathrm{t}$ \\
\hline Inclusão & 3.59 & 0.33 & & 3.48 & 0.37 & 0.920 \\
\hline & & & & & & \\
\hline
\end{tabular}

$\mathrm{Na}$ tabela 4 pode observar-se a correlação existente relativamente à hipótese 3 - "Os pais de crianças NEE com idades mais avançadas experienciam uma atitude negativa face à inclusão que os pais de crianças NEE com idades mais novas".

Encontrou-se um coeficiente de correlação significativo, negativo e moderado entre as atitudes de inclusão e a idade dos filhos com deficiência $(r=-.446)$. Como o coeficiente de correlação é negativo isso significa que quanto mais elevada é a idade do filho com deficiência mais negativa é a atitude de inclusão.

Tabela 4

\begin{tabular}{cc} 
Correlação & \\
\hline & Idade \\
\hline Inclusão & $-.446^{*}$ \\
\hline & $* p \leq .05$
\end{tabular}

Os dados obtido permitiram, ainda, concluir que os pais com níveis mais baixos de escolaridade apresentam 
atitudes significativamente menos favoráveis à inclusão do que os pais com níveis de escolaridade mais elevada (3.39 vs 3.71), $\mathrm{t}(18)=-2.293, p=.034$. o que vem confirmar a hipótese 4 - "Os pais de crianças NEE com baixo nível de escolaridade experienciam uma atitude negativa face à inclusão que os pais de crianças NEE com nível de escolaridade mais alto".

\section{Discussão dos resultados}

As mães, embora a diferença não seja estatisticamente muito significativa, apresentam uma atitude mais favorável à inclusão escolar dos que os pais. Estas conclusões foram confirmadas em parte pelo estudo de Kalyva et al. (2007, cit in Figueiredo, 2015) que apuraram a existência de diferenças nas atitudes inclusivas de acordo com o género, mas na investigação referida foram os pais do sexo masculino que apresentaram atitudes mais inclusivas do que as mães. No entanto, as mães mostraram mais intenção em participar em atividades de interação com os seus filhos e os pares com NEE. Opostamente, no estudo de Barbosa et al. (2007, cit in Figueiredo, 2015, p.62), os resultados obtidos revelaram que os pais tinham atitudes favoráveis à inclusão, independentemente do sexo.

Os pais com níveis de escolaridade mais baixos apresentam atitudes menos favoráveis face à inclusão escolar. Estes resultados foram de encontro às conclusões do estudo de revisão bibliográfica de Boer et al. (2010, cit. in Figueiredo, 2015, p. 63) que constatou que as variáveis estatuto sociocultural e habilitações académicas dos pais estavam relacionadas com as atitudes manifestadas perante a inclusão. Os pais com o estatuto sociocultural mais elevado e habilitações académicas de nível superior tendiam a demonstrar atitudes mais positivas perante a inclusão. No entanto, "resultados obtidos contrariam as conclusões dos estudos de Barbosa et al. (2007), Kalyva et al. (2007) e Tafa e Manolitsis (2003), cujos resultados revelaram que os pais demonstravam atitudes favoráveis à inclusão, independentemente das suas habilitações académicas". (Figueiredo, 2015, p. 63).

\section{Conclusões}

As atitudes dos pais e a forma como percecionam a deficiência dos filhos, parece influenciar de forma positiva ou negativa a inclusão efetiva das crianças com NEE na escola regular.

No panorama educacional vigente, a inclusão escolar constitui um dos grandes desafios do nosso sistema educativo, principalmente na construção e na estruturação de uma escola de todos e para todos, onde todos os alunos, independentemente das suas necessidades e caraterísticas, efetivamente aprendem e têm uma participação ativa na vida escolar/social. Para que isto seja realmente tido em consideração, torna-se essencial a participação e o envolvimento de toda a comunidade educativa. As mudanças só irão ocorrer quando se proporcionarem mais oportunidades de desenvolver práticas educativas inclusivas e as interações entre alunos com e sem NEE forem mais frequentes. Para isso, é necessário que ocorra uma reestruturação das politicas educativas e uma mudança de cultura dentro de cada comunidade escolar. As interações entre as crianças com NEE e a comunidade educativa (alunos, professores, pais, pares) devem ser efetivas e de qualidade, com intuito de desenvolver atitudes mais positivas que auxiliem as mudanças estruturais necessárias ao nível sociocultural.

Os resultados deste estudo sugerem que as atitudes de pais se relacionaram com a perceção da deficiência dos seus filhos e principalmente com o contacto real com a mesma. A escola deverá, também, para além de proporcionar interações de qualidade entre pares com e sem NEE, facultar formações para as famílias dos alunos com e sem NEE, com o objetivo de informar os pais acerca desta realidade, incutindo-lhes os conhecimentos necessários para a compreensão da deficiência, para que estes agentes educativos entendam o significado de inclusão, participando ativamente na construção de uma escola inclusiva.

Neste contexto, considera-se fundamental a realização de mais estudos nesta área que nos proporcione a base teórica para atuar ao nível da formação de atitudes, pois o início da mudança baseia-se nelas. No que diz respeito a futuras investigações, considera-se importante utilizar medidas que privilegiem comparar mães e pais de crianças com NEE com outras mães e pais cujos filhos não apresentam NEE, mas também fazer estudos com outras figuras fundamentais, tais como, os irmãos e mesmo outros prestadores de cuidados, com vista a garantir um maior rigor na extrapolação dos resultados.

\section{Referencias}

Coelho, R. (2010). NEE: Inclusão e relação escola-família. Dissertação de Mestrado apresentada à Escola Superior de Educação de Lisboa, Lisboa, Portugal.

Correia, L.M. (2013). Inclusão e Necessidades Educativas Especiais. Um guia para educadores $e$ professores. Porto: Porto Editora.

Costa, M. (2004). A família com filhos com necessidades educativas especiais. In: Journal of Education, Technologies, and Health, vol.9, n³0, pp.75100

Dessen, M.; Polonia, A. (2007). A Família e a Escola como contextos de desenvolvimento humano. In: Revista Paidéia, vol.17, n³6, pp.21-32

Figueiredo, M. (2015). Atitudes e perceções de pais e filhos perante a inclusão, nas escolas de Angra do Heroísmo. Dissertação de Mestrado apresentada à Universidade Fernando Pessoa, Porto, Portugal.

Matos, E.; Novais, L.; Magalhães, P.; \& Dias, C. (2015). Perceção dos pais em relação à inclusão de crianças com Necessidades Educativas Especiais no ensino regular. In: Revista Educação Especial, vol. 28, $\mathrm{n}^{\circ}$ 52, pp.443-457.

Miranda, P. (2012). Bem-estar psicológico, stress parental, qualidade de vida e suporte social: Um estudo com pais de crianças portadoras de Paralisia Cerebral. Dissertação de Mestrado apresentada à Universidade do Minho, Braga, Portugal. 
Oliveira, T. (2003) A Adaptação Psicossocial dos pais de crianças com Paralisia Cerebral- Estudo Exploratório. Monografia apresentada à Universidade Fernando Pessoa, Porto, Portugal.

UNESCO. (1994). Necessidades Educativas Especiais: Declaração de Salamanca e enquadramento da ação na área das necessidades educativas especiais. Adaptado pela Conferência Mundial da Unesco sobre necessidades educativas especiais. Edição do Instituto de Inovação Educacional, Lisboa. 